\title{
Evaluation of oxidative stress markers and cardiovascular risk factors in Fabry Disease patients
}

\author{
Karen B. Müller ${ }^{1}$, Luciano C. Galdieri ${ }^{1}$, Vanessa G. Pereira ${ }^{1}$, Ana M. Martins ${ }^{1}$ and Vânia D’Almeida ${ }^{1,2}$ \\ ${ }^{1}$ Departamento de Pediatria, Universidade Federal de São Paulo, São Paulo, Brazil. \\ ${ }^{2}$ Departamento de Psicobiologia, Universidade Federal de São Paulo, São Paulo, Brazil.
}

\begin{abstract}
Fabry Disease, an X-linked inborn error of metabolism, is characterized by progressive renal insufficiency, with cardio and cerebrovascular involvement. Homocysteine (Hcy) is considered a risk factor for vascular diseases, but the mechanisms by which it produces cardiovascular damage are still poorly understood. Regarding the vascular involvement in FD patients, the analysis of factors related to thromboembolic events could be useful to improving our understanding of the disease. The aim of this study was to evaluate plasma Hcy and other parameters involved in the methionine cycle, as well as oxidative stress markers. The sample consisted of a group of 10 male FD patients and a control group of 8 healthy individuals, paired by age. Venous blood was collected for Hcy determination, molecular analysis, identification of thiobarbituric acid reactive substances, total glutathione and antioxidant enzymes activity, as well as vitamins quantification. Comparative analysis of FD patients versus the control group indicated hyperhomocysteinemia in 8 of the 10 FD patients, as well as a significant increase in overall glutathione levels and catalase activity. It is inferred that FD patients, apart from activation of the antioxidant system, present increased levels of plasma Hcy, although this is probably unrelated to common alterations in the methionine cycle.
\end{abstract}

Key words: inborn errors of metabolism, Fabry Disease, homocysteine, oxidative stress.

Received: December 16, 2011; Accepted: March 19, 2012.

\section{Introduction}

Fabry Disease (FD) is an X-linked inborn error of glycosphingolipid metabolism caused by deficient lysosomal enzyme $\alpha$-galactosidase A activity. As a result, neutral glycosphingolipid globotriaosylceramide (Gb3) accumulates in the liver, heart, spleen and kidneys, especially in lysosomes of vascular endothelium and plasma (Desnick et al., 2001; Desnick et al., 2003).

The disease is pan-ethnic, with the incidence of classical phenotypes estimated to be around 1:40,000, although a study with a newborn screening sample has found an incidence of 1:3,100 (Spada et al., 2006). FD predominantly affects males (hemizygous), although carrier (heterozygous) females can also be affected to a mild or even severe extent (Whybra et al., 2001; Desnick et al., 2003). FD is characterized by progressive renal insufficiency, leading to morbidity through cardio- and cerebrovascular involvement. Major manifestations include paresthesia in the extremities, corneal dystrophy, angiokeratoma and occlusive vascular disease of the heart, kidney and brain, leading to premature death.

Send correspondence to Vânia D'Almeida. Departamento de Psicobiologia, Universidade Federal de São Paulo, Rua Napoleão de Barros 925, Vila Clementino, 04024-002 São Paulo, SP, Brazil. E-mail: vaniadalmeida@uol.com.br.
Apart from thrombogenic factors, which can also play a significant role in FD vascular pathology (Demuth and Germain, 2002), FD patients present an increased incidence of arterial thrombosis, mainly due to the progressive deposition of $\mathrm{Gb} 3$ in vascular endothelium and smooth muscle cells (Hughes and Mehta, 2005).

Whereas homocysteine (Hcy), a sulfur containing amino acid formed during methionine metabolism, is considered by some authors as a risk factor in vascular diseases (Refsum et al., 1998; Homocysteine Studies Collaboration, 2002; Lentz and Haynes, 2004), hyperhomocysteinemia (HHcy), at a plasmatic concentration higher than $15 \mu \mathrm{mol} / \mathrm{L}$ has also been associated with disorders, such as venous thrombosis, ischemic heart disease, atherothrombosis and strokes (Morelli et al., 2002; Lentz and Haynes, 2004; Eldibany and Caprini, 2007).

Although the mechanisms by which Hcy induces cardiovascular damage are still poorly understood, those known include endothelial dysfunction, and the oxidative stress process (Welch and Loscalzo, 1998; Voutilainen et al., 1999; Sharma et al., 2006). Cysteine (Cys) (Homocysteine Studies Collaboration, 2002), another sulfur containing amino acid involved in methionine metabolism, has also been associated with oxidative stress conditions (Jones, 2006). 
These oxidative stress conditions, defined by disruption of the physiological balance between oxidants and antioxidants, potentially lead to cellular damage. Some authors propose that Hcy induced oxidative stress is primarily caused by detoxification and intracellular antioxidant enzyme deficiency (Sharma et al., 2006).

Oxidative stress seems to play a role in the pathophysiology of several inborn errors of metabolism, such as phenylketonuria, maple syrup disease and homocystinuria (Ristoff and Larsson, 2002; Sirtori et al., 2005; Barschak et al., 2006). An increase in the antioxidant enzyme activity catalase (CAT) has been observed in two other lysosomal storage disorders, Gaucher Disease and Mucopolysaccharidosis type I (Roversi et al.,2006; Pereira et al., 2008).

Considering that vascular involvement is an important characteristic in FD patients, the aim here was to evaluate total plasma concentrations of Hcy and Cys, other factors involved in the methionine cycle (vitamin B12, folic acid and the C677T polymorphism of methylenetetrahydrofolate reductase - MTHFR), and oxidative stress markers, i.e., total glutathione, lipid peroxidation and antioxidant enzymes (CAT, superoxide dismutase - SOD and glutathione peroxidase - GPx), to so be able to evaluate the role of these parameters in FD patients.

\section{Material and Methods}

\section{Subjects}

The sample consisted of two groups: 10 male FD patients, from 16 to 70 years old (termed FD group), and eight healthy male volunteers, from 18 to 60 years old (termed control group - CG). Patients were selected from the $1^{\circ}$ Encontro Brasileiro de Familiares e Portadores da Doença de Fabry (1st Brazilian meeting of Fabry disease patients and relatives), bringing together patients and relatives countrywide, and which took place at the Univer- sidade Federal de São Paulo (UNIFESP). Healthy volunteers were selected from among the UNIFESP staff.

All the FD patients participated voluntarily, after having given written informed consent. Diagnosis was confirmed by enzymatic activity assay and/or molecular analysis. Besides kinship (three pairs of siblings), six individuals were undergoing enzyme replacement therapy at the time of the study (Table 1).

The study took place at the Centro de Referência em Erros Inatos do Metabolismo (CREIM). The research protocols and consent forms, as well as the overall investigation, were ethically and scientifically approved by the Medical Research and Ethical Committee of UNIFESP.

Venous blood from each patient was collected in three different tubes, one containing EDTA, another heparin and the third without anticoagulant. Leukocytes and plasma from the EDTA tubes were separated by centrifugation at $1000 \mathrm{xg}$ for $10 \mathrm{~min}$. Plasma was used for Hcy and Cys quantification, and leukocytes for DNA extraction prior to polymorphism analysis. Red blood cells (RBC) and plasma from tubes containing heparin were separated by centrifugation at $1000 \mathrm{xg}$ for $6 \mathrm{~min}$ at $4{ }^{\circ} \mathrm{C}$. The plasma was immediately used for lipid peroxidation analysis, whereas the RBCs were stored at $-80{ }^{\circ} \mathrm{C}$ for posterior total glutathione and antioxidant enzymes assay. A hemolysate of $\mathrm{RBC}$ for each sample was prepared by washing and lysing these cells.

An aliquot of these hemolysates was used for antioxidant enzymes assay, and another mixed with $\mathrm{HClO}_{4} 2 \mathrm{M}$ EDTA $4 \mathrm{mM}$ for the analysis of total glutathione (tGSH). Serum separated from tubes without anticoagulant was used for vitamin B12 and folic acid quantification. Urine samples were collected from eight patients and stored at $-80{ }^{\circ} \mathrm{C}$ prior to analysis of Hcy and Cys excretion (Cyanide-Nitroprusside test).

Table 1 - Characteristics of the Fabry Disease patients.

\begin{tabular}{|c|c|c|c|c|c|c|c|c|c|}
\hline FD patients & Age & $\mathrm{ERT}^{\#}$ & Relationship & Angiokeratoma & $\begin{array}{c}\text { Cornea } \\
\text { verticilata }\end{array}$ & Acroparestesia & Hypohidrosis & Proteinuria & $\begin{array}{l}\text { Vascular } \\
\text { events }\end{array}$ \\
\hline 1 & 26 & NO & $2 ; 5$ & + & $\mathrm{x}$ & + & + & + & YES \\
\hline 2 & 31 & 5 & $1 ; 5$ & + & $\mathrm{x}$ & + & + & + & NO \\
\hline 3 & 33 & 10 & & + & $\mathrm{x}$ & + & + & + & NO \\
\hline 4 & 47 & 2 & 12 & + & + & + & + & + & NO \\
\hline 6 & 73 & NO & & + & + & + & + & + & NO \\
\hline 10 & 20 & NO & 7 & & & & & & NO \\
\hline 11 & 46 & NO & & + & + & + & + & + & NO \\
\hline 12 & 46 & 2 & 4 & + & - & + & + & + & NO \\
\hline 14 & 36 & 2 & $9 ; 13 ; 15 ; 16$ & + & + & + & + & + & NO \\
\hline 16 & 49 & 5 & $9 ; 13 ; 14 ; 15$ & $\mathrm{x}$ & + & + & + & + & NO \\
\hline
\end{tabular}

All the symptoms were obtained from the Fabry Registry (patient 10 not included in Fabry Registry); +: manifestation present; -: manifestation not occurs; $x$ : data not accessed. Age (years) at the time of sample collection. ERT: enzyme replacement therapy; \#: time in months until the collection. ${ }^{\dagger}$ : data until 2007 (from the Fabry Registry). 


\section{Biochemical analysis}

Thiobarbituric acid reactive substances (TBARS) were quantified in plasma for lipid peroxidation analysis (Ohkawa et al., 1979). Results were expressed as nmol of malondialdehyde/mL. The tGSH assay was carried out according to the method described by Tietze (1969). Samples were analyzed spectrophotometrically and results expressed as $\mu \mathrm{mol} / \mathrm{g}$ hemoglobin (Hb). CAT activity assay was according to the method described by Adamo et al. (1989). Activity values were expressed as units per milligram of hemoglobin (U/mg Hb). GPx activity levels were measured using the method described by Sies et al. (1979), and the results expressed as U/g Hb. SOD activity was assayed using the method described by McCord and Fridovich (1969), and the result calculated as U/mg Hb.

Evaluation of Hcy and Cys concentrations in plasma, according to the method described by Guerra-Shinohara et al. (2002), was performed with high performance liquid chromatography. Concentrations were given as $\mu \mathrm{M}$. Quantification of vitamin B12 was performed by immunoassay with the Roche Diagnostics kit (Elecsys ${ }^{\circledR} 2010$ - Vitamin B12). Folic acid was quantified by HPLC(Kelly and Davis, 1964). Concentrations of both vitamins were given as $\mathrm{pg} / \mathrm{mL}$ and $\mathrm{ng} / \mathrm{mL}$, respectively.

Cyanide-Nitroprusside testing was performed according to the method described by Shih et al. (1991).

\section{Molecular analysis}

Genomic DNA was obtained from peripheral blood samples, according to the method described by Miller et al. (1988). Analysis of MTHFR gene C677T polymorphism was by polymerase chain reaction (PCR) and restriction fragment length polymorphism analysis. Primers were described by Goyette et al. (1995). The final PCR product was digested with the restriction enzyme Hinf I.

\section{Statistical analysis}

Data were expressed as means \pm standard deviation (SD). The Student $t$ test for two independent samples was applied for analyzing the means of FD patients versus CG. The significance level was set at five percent. All the analyses were with STATISTICA 6.0 for Windows software.

\section{Results}

The Fabry patients clinical and demographic data (Table 1) were obtained from questionnaires applied during the day of sample collection, and supplemented by information from the Fabry Registry sponsored by the Genzyme Corporation.

All the biochemical parameters in the 10 male FD patients were analyzed, and the results subsequently compared to data obtained from $\mathrm{CG}(\mathrm{n}=8$ male volunteers). Regarding Hcy levels, the average result observed in the FD group was significantly higher than that observed in CG
( $\mathrm{p}=0.0093$; FD group: $22.20 \pm 8.40$ - without outlier; CG: $11.35 \pm 2.82)$. Worthy of note, one of the patients presented a much higher Hcy value than the average (252.64), thereby justifying exclusion from analysis. On applying the definition of HHcy, a significant increase could be observed in eight of the 10 FD patients (Figure 1). Cys quantification, applied to FD patients and CG alike, indicated no apparent difference between the two groups ( $\mathrm{p}=0.6494$; FD group: $508.60 \pm 92.46$; CG: $485.41 \pm 110.54$ ).

With the exception of three patients with B12 levels below the normal range (Vitamin B12: $228.60 \pm 89.77$ $\mathrm{pg} / \mathrm{mL}$ - reference: $180-950 \mathrm{pg} / \mathrm{mL}$; Folic acid: $6.37 \pm 0.89 \mathrm{ng} / \mathrm{mL}$ - reference: $1.8-9.0 \mathrm{ng} / \mathrm{mL}$ ), vitamin B12 and folic acid quantification with FD group samples presented averages for both parameters, which could be considered as in accordance with reference values obtained from the laboratory responsible for determination (VITAE Cromatografia Líquida em Análises Clínicas LTDA, São Paulo, Brazil).

MTHFR C677T polymorphism was observed in five of the FD patients. Concerning oxidative stress parameters, a significant increase in tGSH levels and CAT activity was observed, when compared to CG (Table 2).

\section{Discussion}

Plasma Hcy levels higher than $15 \mu \mathrm{mol} / \mathrm{L}$ were observed in $80 \%$ of the FD samples. According to the literature, in the population as a whole, there are many reasons for this increase, such as vitamin deficiency and mutation in those genes that encode enzymes from the methionine cy-

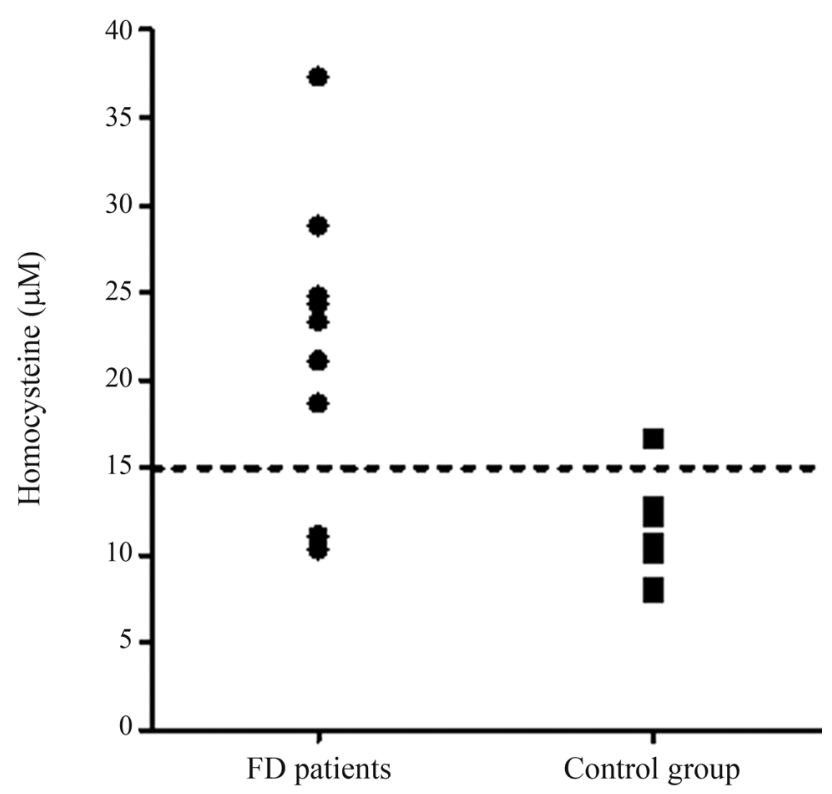

Figure 1 - Homocysteine concentration values in FD and CG. Data are from FD patients, but excluding the outlier value $(252.64 \mu \mathrm{M})$. An asterisk indicates statistically significant differences between FD patients and CG $(\mathrm{p}=0.0093)$. The shaded area represents the normal Hcy concentration. 
Table 2 - Oxidative stress data obtained from FD patients and CG.

\begin{tabular}{lcc}
\hline & Fabry Disease patients & Control group \\
\hline Total Glutathione (mmol/g Hb) & $6.37 \pm 0.89^{*}$ & $4.77 \pm 0.94$ \\
Lipid peroxidation (nmol MDA/mL) & $1.13 \pm 0.46$ & $1.41 \pm 0.39$ \\
Catalase (U/mg Hb) & $97.02 \pm 17.47^{*}$ & $73.04 \pm 22.66$ \\
Superoxide Dismutase (U/mg Hb) & $15.79 \pm 3.35$ & $15.74 \pm 3.23$ \\
Glutathione Peroxidase (mU/mg Hb) & $11.08 \pm 0.90$ & $10.60 \pm 5.16$ \\
\hline
\end{tabular}

Data are expressed in mean $+/$ SD. Statistical $\mathrm{p}$ values were obtained with the Student $\mathrm{t}$ test. ${ }^{*} \mathrm{p}$ value $<0.05$.

cle, being among the most frequent (Lentz and Haynes, 2004; Eldibany and Caprini, 2007).

Quantification of vitamin B12 and folic acid, as well as analysis of MTHFR C677T polymorphism, were used to assess any relationship between alterations in these parameters and HHcy. Although the average levels of both vitamins in FD patients were in accordance with the reference value range, three patients presented low vitamin B12 levels. Considering that these three patients presented HHcy, vitamin alteration could probably be responsible for this. Since vitamin B12 deficiency has not been observed previously among FD patients (Sheth et al., 1981; Fedi et al., 2005), our findings deserves consideration. As to MTHFR polymorphisms, it was impossible to establish a direct relationship between the presence of the T allele and HHcy. Of the five unrelated patients found to be heterozygous for this allele, only three presented HHcy.

Since an increase in plasma Hcy levels was noted, it was decided to check for any parallel increase in Hcy excretion, a characteristic of patients with homocystinuria. As the Cyanide-Nitroprusside test shows the presence of sulfur-containing amino acids in urine, this was applied to analyze both Hcy and Cys excretion. No alteration was found in the urine from the six FD patients presenting HHcy. As the kidney is essential for Hcy clearance and metabolism, it is possible that the renal damage observed in FD patients contributes to the imbalance of Hcy levels (Friedman et al., 2001).

It is important to describe one particular case observed during the study. One subject presented a very high Hcy level $(252.64 \mu \mathrm{M})$, but with no increase in Hcy and/or Cys excretion detectable by the Cyanide-Nitroprusside test. Although the patient presented vitamin B12 levels below the normal range and was heterozygous for MTHFR gene C677T polymorphism, these factors could not account for such a high Hcy level. Hence, there is the possibility of this alteration arising from renal damage, since the individual presented no symptoms of homocystinuria. Nevertheless, Hcy levels $>200 \mu \mathrm{M}$ are compatible with this inborn error of metabolism (Refsum et al., 1998).

The relationship between HHcy and increased cardiovascular risk is widely known (Refsum et al., 1998; Welch and Loscalzo, 1998; Homocysteine Studies Collaboration, 2002). In a study published in 2002, an increase in Hcy lev- els was observed in FD patients (Demuth and Germain, 2002), yet the authors did not include subjects with kidney insufficiency, and failed to supply pertinent information regarding more premature stages of renal damage. HHcy was also observed when comparing FD patients versus CG, although in this case the decrease in vitamin B6 and folic acid levels was considered to account for the alteration (Fedi et al., 2005). One limitation in our study was the lack of information regarding renal function in the patients, thus limiting any assessment of the possible relationship between HHcy and renal failure.

There was no significant difference in TBARS plasma levels, a marker of lipid peroxidation, and thus indicative of oxidative stress. However, an increase in tGSH levels and CAT activity was observed among FD patients, thereby implying activation of the antioxidant system.

A similar activation of antioxidant defense in Gaucher disease and Mucopolysaccharidosis type 1 (MPS 1) patients, had already been demonstrated by our group (Roversi et al., 2006; Pereira et al., 2008). Furthermore, an increase in CAT activity and a decrease in SOD activity had been observed in Gaucher Disease patients undergoing enzyme replacement therapy (ERT) (Roversi et al., 2006). Upon evaluating MPS I patients before starting ERT and up to 24 weeks of infusion, the increase in CAT activity was seen to occur after the beginning of the treatment, thereby indicating that activation of the antioxidant system may be a direct consequence of ERT (Pereira et al., 2008).

Hence, as $60 \%$ of the FD group was composed of patients undergoing ERT, activation of antioxidant defense observed among these may also be associated to the treatment. Corroborating this hypothesis, a recent study (Shen et al., 2008) demonstrated that incubation of cells with recombinant $\alpha$-galactosidase A led to an increase in the production of reactive oxygen species (ROS), thus in accordance with the previous results from our group (Roversi et al., 2006; Pereira et al., 2008). Furthermore, it was demonstrated that $\mathrm{Gb} 3$ also induces the increased production of ROSs, when added to the cell culture medium. This excessive ROS generation apparently being linked with the involvement of vascular endothelial cells, resulting in the development of atherosclerosis and other cardiovascular disorders frequently encountered in FD patients. 
In conclusion, our results suggest that FD patients present increased levels of plasma Hcy, probably unrelated to common alterations in the methionine cycle, as well as activation of the antioxidant system.

\section{Acknowledgments}

This work was supported by the Conselho Nacional de Desenvolvimento Científico e Tecnológico/CNPq Brazil (process\#473924/2003-0), Fundação de Amparo à Pesquisa do Estado de São Paulo/FAPESP - Brazil (process\#97/1870-4 and 01/11366-9), Associação Fundo de Incentivo a Psicofarmacologia/AFIP, and Instituto de Genética e Erros Inatos do Metabolismo (IGEIM). We also thank $\mathrm{CNPq}$ for a fellowship granted to our technical staff (process\#501248/2005-6). V.D'A. is recipient of CNPq fellowship. The authors are also grateful for the technical assistance provided by VITAE Cromatografia Líquida em Análises Clínicas LTDA, and to Allan Chiaratti de Oliveira, Mayra Del Bosco Rodrigues and Cecília Micheletti.

\section{References}

Adamo AM, Llesuy SF, Pasquini JM and Boveris A (1989) Brain chemiluminescence and oxidative stress in hyperthyroid rats. Biochem J 263:273-277.

Barschak AG, Sitta A, Deon M, de Oliveira MH, Haeser A, Dutra-Filho CS, Wajner M and Vargas CR (2006) Evidence that oxidative stress is increased in plasma from patients with maple syrup urine disease. Metab Brain Dis 21:279286.

Demuth K and Germain DP (2002) Endothelial markers and homocysteine in patients with classic Fabry disease. Acta Paediatr Suppl 91:57-61.

Desnick RJ, Ioannou YA and Eng CM (2001) Alpha-galactosidase A deficiency: Fabry Disease. In: Scriver CR, Beaudet AL, Sly WS and Valle D (eds) The Metabolic and Molecular Bases of Inherited Disease. 8th edition. McGraw-Hill, New York, $6338 \mathrm{pp}$.

Desnick RJ, Brady R, Barranger J, Collins AJ, Germain DP, Goldman M, Grabowski G, Packman S and Wilcox WR (2003) Fabry disease, an under-recognized multisystemic disorder: Expert recommendations for diagnosis, management, and enzyme replacement therapy. Ann Intern Med 138:338-346.

Eldibany MM and Caprini JA (2007) Hyperhomocysteinemia and thrombosis: An overview. Arch Pathol Lab Med 131:872884.

Fedi S, Gensini F, Gori AM, Abbate R and Borsini W (2005) Homocysteine and tissue factor pathway inhibitor levels in patients with Fabry's disease. J Thromb Haemost 3:21172119.

Friedman AN, Bostom AG, Selhub J, Levey AS and Rosenberg IH (2001) The kidney and homocysteine metabolism. J Am Soc Nephrol 12:2181-2189.

Goyette P, Frosst P, Rosenblatt DS and Rozen R (1995) Seven novel mutations in the methylenetetrahydrofolate reductase gene and genotype/phenotype correlations in severe methylenetetrahydrofolate reductase deficiency. Am J Hum Genet 56:1052-1059.
Guerra-Shinohara EM, Paiva AA, Rondo PH, Yamasaki K, Terzi CA and D'Almeida V (2002) Relationship between total homocysteine and folate levels in pregnant women and their newborn babies according to maternal serum levels of vitamin B12. BJOG 109:784-791.

Homocysteine Studies Collaboration (2002) Homocysteine and risk of ischemic heart disease and stroke: A meta-analysis. JAMA 288:2015-2022.

Hughes DA and Mehta AB (2005) Vascular complications of Fabry disease: Enzyme replacement and other therapies. Acta Paediatr 94(Suppl):28-33 (see also Discussion pp 9$10)$.

Jones DP(2006) Redefining oxidative stress. Antioxid Redox Signal 8:1865-1879.

Kelly A and Davis RE (1964) Serum and liver folate activity in the rat. Aust J Exp Biol Med Sci 42:209-214.

Lentz SR and Haynes WG (2004) Homocysteine: Is it a clinically important cardiovascular risk factor? Cleve Clin J Med 71:729-734.

McCord JM and Fridovich I (1969) Superoxide dismutase - an enzymic function for erythrocuprein (hemocuprein). J Biol Chem 244:6049-6055.

Miller SA, Dykes DD and Polesky HF (1988) A simple salting out procedure for extracting DNA from human nucleated cells. Nucleic Acids Res 16:1215.

Morelli VM, Lourenco DM, D'Almeida V, Franco RF, Miranda F, Zago MA, Noguti MA, Cruz E and Kerbauy J (2002) Hyperhomocysteinemia increases the risk of venous thrombosis independent of the C677T mutation of the methylenetetrahydrofolate reductase gene in selected Brazilian patients. Blood Coagul Fibrinolysis 13:271-275.

Ohkawa H, Ohishi N and Yagi K (1979) Assay for lipid peroxides in animal tissues by thiobarbituric acid reaction. Anal Biochem 95:351-358.

Pereira VG, Martins AM, Micheletti C and D'Almeida V (2008) Mutational and oxidative stress analysis in patients with mucopolysaccharidosis type I undergoing enzyme replacement therapy. Clin Chim Acta 387:75-79.

Refsum H, Ueland PM, Nygard O and Vollset, SE (1998) Homocysteine and cardiovascular disease. Annu Rev Med 49:3162.

Ristoff E and Larsson A (2002) Oxidative stress in inborn errors of metabolism: Lessons from glutathione deficiency. J Inherit Metab Dis 25:223-226.

Roversi FM, Galdieri LC, Souza FG, Micheletti C, Martins AM and D'Almeida V (2006) Blood oxidative stress markers in Gaucher disease patients. Clin Chim Acta 364:316-320.

Sharma P, Senthilkumar RD, Brahmachari V, Sundaramoorthy E, Mahajan A, Sharma A and Sengupta S (2006) Mining literature for a comprehensive pathway analysis: A case study for retrieval of homocysteine related genes for genetic and epigenetic studies. Lipids Health Dis 5:e1.

Shen JS, Meng XL, Moore DF, Quirk JM, Shayman JA, Schiffmann R and Kaneski CR (2008) Globotriaosylceramide induces oxidative stress and up-regulates cell adhesion molecule expression in Fabry disease endothelial cells. Mol Genet Metab 95:163-168.

Sheth KJ, Werlin SL, Freeman ME and Hodach AE (1981) Gastrointestinal structure and function in Fabry's disease. Am J Gastroenterol 76:246-251. 
Shih VE, Mandell R and Sheinhait I (1991) General metabolic screening tests. In: Hommes FA (ed) Techniques in Diagnostic Human Biochemical Genetics - A Laboratory Manual. 1st edition. Wiley-Liss, New York, pp 49-50.

Sies H, Koch OR, Martino E and Boveris A (1979) Increased biliary glutathione disulfide release in chronically ethanoltreated rats. FEBS Lett 103:287-290.

Sirtori LR, Dutra-Filho CS, Fitarelli D, Sitta A, Haeser A, Barschak AG, Wajner M, Coelho DM, Llesuy S, Bello-Klein A, et al. (2005) Oxidative stress in patients with phenylketonuria. Biochim Biophys Acta 1740:68-73.

Spada M, Pagliardini S, Yasuda M, Tukel T, Thiagarajan G, Sakuraba H, Ponzone A and Desnick RJ (2006) High incidence of later-onset Fabry disease revealed by newborn screening. Am J Hum Genet 79:31-40.

Tietze F (1969) Enzymic method for quantitative determination of nanogram amounts of total and oxidized glutathione: Appli- cations to mammalian blood and other tissues. Anal Biochem 27:502-522.

Voutilainen S, Morrow JD, Roberts $2^{\text {nd }}$ LJ, Alfthan G, Alho H, Nyyssonen K and Salonen JT (1999) Enhanced in vivo lipid peroxidation at elevated plasma total homocysteine levels. Arterioscler Thromb Vasc Biol 19:1263-1266.

Welch GN and Loscalzo J (1998) Homocysteine and atherothrombosis. N Engl J Med 338:1042-1050.

Whybra C, Wendrich K, Ries M, Gal A and Beck M (2001) Clinical manifestation in female Fabry disease patients. Contrib Nephrol 136:245-250.

Associate Editor: Mara Hutz

License information: This is an open-access article distributed under the terms of the Creative Commons Attribution License, which permits unrestricted use, distribution, and reproduction in any medium, provided the original work is properly cited. 